\title{
DISTRIBUCIÓN ACTUAL, POTENCIAL Y ECOFISIOGRÁFICA DE ESPECIES DE CALABAZA EN NAYARIT, MÉXICO
}

\section{CURRENT, POTENTIAL AND ECOPHYSIOGRAPHICAL DISTRIBUTION OF PUMPKIN SPECIES IN NAYARIT, MEXICO}

\author{
Roberto Valdivia-Bernal ${ }^{1}$, Karina S. Vera-Sánchez ${ }^{2}$, \\ Pablo G. Ruelas-Hernández ${ }^{1}$ * y Beatriz G. Arrieta-Ramos ${ }^{1}$
}

\begin{abstract}
'Universidad Autónoma de Nayarit, Unidad Académica de Agricultura, Xalisco, Nayarit, México. ${ }^{2}$ Universidad Autónoma Chapingo, Instituto de Horticultura, Chapingo, Texcoco, Estado de México, México.

*Autor de correspondencia (pablogerman82@hotmail.com)
\end{abstract}

\section{RESUMEN}

La calabaza (Cucurbita spp.) es un género originario de América, del cual algunas especies importantes son originarias de México, tiene arraigo cultural y es utilizada en la alimentación humana y animal, por lo que su aprovechamiento, mejoramiento y conservación son esenciales. El objetivo de esta investigación fue generar mapas de distribución actual y potencial de especies de calabaza cultivadas y silvestres, así como precisar su distribución ecofisiográfica en el estado de Nayarit, México. Se utilizaron 114 registros de sitios georreferenciados de especies domesticadas (Cucurbita moschata, $C$. pepo, $C$. argyrosperma y $C$. ficifolia) y silvestres (C. radicans, $C$. martinezii) provenientes de 66 accesiones y 48 registros de herbarios. Se emplearon 110 variables bioclimáticas, geofísicas y edáficas. Para su análisis se utilizaron herramientas de sistemas de información geográfica (SIG) y procedimientos multivariados de componentes principales (CP) y de conglomerados. La distribución actual mostró que las especies de calabazas se encuentran presentes en las cuatro regiones fisiográficas del estado de Nayarit, entre alturas de 7 a $2117 \mathrm{msnm}$. La distribución potencial permitió la ubicación de sitios no registrados con probable presencia de calabazas. En la distribución ecofisiográfica, tres $\mathrm{CP}$ representaron el $77 \%$ de la variabilidad total, las variables que mostraron mayor correlación con el CP1 fueron altitud (m) y temperatura $\left({ }^{\circ} \mathrm{C}\right)$, con el $\mathrm{CP} 2$ precipitación $(\mathrm{mm})$, características de suelo (textura, profundidad y $\mathrm{pH}$ ) y velocidad del viento $\left(\mathrm{km} \mathrm{h}^{-1}\right)$ y con el CP3 precipitación de los tres meses más fríos del año. En especies de calabaza esta información permitirá ubicar áreas de probable presencia para exploraciones más dirigidas, conocer las condiciones ambientales de sus hábitats y utilizarla en futuros programas de conservación.

Palabras clave: Cucurbita spp., calabaza, distribución ecofisiográfica, distribución de especies, sistemas de información geográfica (SIG), variables bioclimáticas.

\section{SUMMARY}

Pumpkin (Cucurbita spp.) is a genus originating in America. Some important pumpkin species originated in Mexico, and it has cultural roots and uses as human food and animal feed. Thus, its use, improvement, and conservation are essential. This research generated maps of the current and potential distribution of cultivated and wild species of pumpkin and identified their eco-physiographic distribution in the state of Nayarit, Mexico. One hundred fourteen records from georeferenced sites of domesticated (Cucurbita moschata, $C$. pepo, $C$. argyrosperma, and $C$. ficifolia) and wild species $(C$. radicans, $C$. martinezii) from 66 accessions and 48 herbarium records were used. One hundred and ten bioclimatic, geophysical, and edaphic variables were also used. Geographic information systems (GIS) tools and multivariate procedures, like principal component (PC) and cluster analyses were employed for the study. The current distribution showed that these pumpkin species are present in the four physiographic regions of the state of Nayarit, at altitudes ranging from 7 to 2117 masl. The potential distribution allowed the location of unregistered sites with probable presence of pumpkin. In the ecophysiographic distribution, three PC represented $77 \%$ of the total variability: altitude $(\mathrm{m})$ and temperature $\left({ }^{\circ} \mathrm{C}\right)$ showed the highest correlation with $\mathrm{CP} 1$; rainfall $(\mathrm{mm})$, soil characteristics (texture, depth, and $\mathrm{pH}$ ) and wind speed $\left(\mathrm{km} \mathrm{h}^{-1}\right)$ correlated with CP2; and precipitation in the three coldest months of the year associated with CP3. In pumpkin species, this information will allow the identification of areas of probable presence for targeted explorations that recognize the environmental conditions of these habitats, and that information might be used in future conservation programs.

Index words: Cucurbita spp., bioclimatic variables, ecophysiographic distribution, geographic information systems (GIS), species distribution, squash.

\section{INTRODUCCIÓN}

Las especies de Cucurbita se encuentran entre las plantas cultivadas más antiguas de América (Whitaker y Bohn, 1950) y junto con el maíz, frijol, chile, amaranto y vainilla constituye uno de los 26 cultivos de mayor importancia económica en el mundo (Mastretta-Yanes et al., 2019). La calabaza fue la primera planta cultivada en Mesoamérica, su fecha de domesticación data de hace unos 10,000 años (Smith, 1997). Las calabazas que se consumen en el mundo fueron obtenidas de especies que fueron domesticadas en México, se utilizan en la alimentación como verdura fresca y el fruto, tierno o maduro, en la repostería. Sus semillas son aprovechadas tostadas para consumo directo o empleadas como complemento en la elaboración de moles o pipianes y son un guiso tradicional en nuestro país (Villanueva, 2007). 
Siempre ha sido un tema de interés el análisis de la relación entre las especies y su hábitat (Seoane y Bustamante, 2001); el área de distribución de una especie sugiere la presencia de ésta en un espacio geográfico (Maciel-Mata et al., 2015). Los modelos de distribución de especies son representaciones cartográficas de la idoneidad de un espacio para la presencia de éstas en función de las variables empleadas para generar dicha representación (Mateo et al., 2011).

El análisis espacial puede contribuir significativamente a una mejor comprensión y monitoreo de la biodiversidad. Los resultados del análisis espacial permiten la formulación y la implementación de estrategias de conservación más específicas para evaluar el estado que guardan las especies de plantas y priorizar áreas para la conservación (Scheldeman y van Zonneveld, 2010).

Los datos que provienen de los recursos genéticos pueden ser analizados en conjunción con datos georreferenciados, y además se puede utilizar información del clima, vegetación y suelo de la región de estudio para estimar la distribución y la fenología de un taxón. Este tipo de análisis se lleva a cabo utilizando herramientas de sistemas de información geográfica (SIG) (Guarino et al., 2002).

En el estado de Nayarit el cultivo de calabaza (Cucurbita spp.) se realiza en la mayor parte de su territorio, ampliamente cambiante en su fisiografía, lo cual lo hace poseedor de una variedad de ambientes naturales que contribuyen a la presencia de una gran diversidad de especies vegetales y animales; la documentación de la distribución de calabazas mediante sistemas de información geográfica (SIG) es importante para propósitos de planificación para la conservación de especies, aprovechamiento y mejoramiento, por lo que el objetivo del presente estudio fue determinar la distribución geográfica actual, potencial y ecofisiográfica de especies de calabaza en el estado de Nayarit.

\section{MATERIALES Y MÉTODOS}

\section{Especies en estudio}

La información se obtuvo de datos pasaporte de 114 puntos geográficos donde se han colectado especies de Cucurbita spp. en el estado de Nayarit. Incluye información de 48 registros de herbario (GBIF, 2018) y sitios de 66 accesiones de los años 2012 a 2016 (Cuadro 1); previo al presente estudio se realizó una caracterización morfólogica (Ruelas et al., 2015) y documentación (datos pasaporte), y parte del germoplasma fue enviado al Sistema Nacional de Recursos Fitogenéticos (SINAREFI) para su conservación.

\section{Distribución actual y modelación de la distribución potencial}

Con el fin de determinar la calidad de georeferenciación de los sitios de recolección y registros de especies de calabaza, se aplicó la herramienta GEOQUAL de CAPFITOGEN 2.0 (Parra-Quijano et al., 2015) descartando de los posteriores análisis espaciales aquellos sitios con una calidad inferior a 80 en una escala de 0 a 100. Con base en estos datos, y de acuerdo con Scheldeman y van Zonneveld (2010), se realizó una modelación de la distribución potencial y actual para cada especie del género Cucurbita, mediante Maxent y DIVA-GIS versión 7.5.0 con capas ráster en un sistema de coordenadas geográficas WGS-84 y celdas de 2.5 arc$\min (5 \times 5 \mathrm{~km}$ del ecuador). La edición de los mapas se realizó con ArcMap 10.2.2. (ESRI, 2014), donde las celdas con valores más cercanos a 1 indican alta probabilidad de que se encuentren las especies del género Cucurbita spp. Esta modelación se basó en 19 parámetros climáticos relacionados con temperatura y precipitación de la base de datos climáticos Worldclim (http://www.worldclim.org/ bioclim), consultada en abril de 2018.

\section{Distribución ecofisiográfica}

Esta distribución se obtuvo de cada uno de los 114 sitios de recolección y registro de Cucurbita spp. con la aplicación de la herramienta ECOGEO y CAPFITOGEN 2.0 (ParraQuijano et al., 2015). Se construyó una matriz utilizando 110 variables: 1) geofísicas: Alt: altura (msnm), srad_1-12: radiación solar mensual $\left(\mathrm{W} \mathrm{m}^{-2}\right)$, wind_1-12: velocidad del viento mensual $\left(\mathrm{km} \mathrm{h}^{-1}\right)$, prec_1-12: precipitación mensual (mm), t_max, t_min, t_mean: temperatura máxima, mínima y media mensual $\left({ }^{\circ} \mathrm{C}\right)$; 2) bioclimáticas: bio_1-19: variables relacionadas con temperatura $\left({ }^{\circ} \mathrm{C}\right)$ y precipitación $\left.(\mathrm{mm}) ; 3\right)$ edáficas: t_clay: arcilla (\%), t_sand: arena (\%), t_teb: bases intercambiables, t_caco3: carbonatos, t_ph_h2o: pH, ref_ depth: profundidad suelo $(\mathrm{m})$. Con estos datos se realizó un análisis multivariado de componentes principales y de agrupamiento por el método de Ward. Los análisis se realizaron con el programa SAS versión 8 (SAS Institute, 1999) y el Programa Estadístico Minitab ${ }^{\circledR} 15$ (Minitab, 2007)

\section{RESULTADOS Y DISCUSIÓN}

\section{Distribución actual}

Considerando las cuatro regiones fisiográficas de Nayarit (Llanura Costera del Pacifico, Eje Neovolcánico, Sierra Madre del Sur y Sierra Madre Occidental) (Figura 1A), en el estado se distribuyen seis especies de calabaza, cuatro domesticadas (C. argyrosperma, C. moschata, C. pepo y C. ficifolia) (Ruelas et al., 2015) y dos silvestres 
Cuadro 1. Registros y número de accesiones de calabaza (Cucurbita spp.) distribuidas en el estado de Nayarit, México.

\begin{tabular}{|c|c|c|c|c|}
\hline Especie & Registros de herbario & $\begin{array}{l}\text { Número de sitios de } \\
\text { recolectas }\end{array}$ & Total de registros & $\begin{array}{c}\text { Intervalo altitudinal } \\
\text { (msnm) }\end{array}$ \\
\hline C. moschata & 10 & 21 & 31 & $11-1347$ \\
\hline C. argyrosperma & 31 & 24 & 55 & $7-1925$ \\
\hline C. ficifolia & & 7 & 7 & $1705-2117$ \\
\hline C. pepo & 2 & 7 & 9 & $1051-2117$ \\
\hline C. radicans & 2 & & 2 & $1040-1215$ \\
\hline C. martinezii & & 3 & 3 & $55-418$ \\
\hline${ }^{\dagger} \mathrm{C} . \mathrm{sp}$. & 3 & 4 & 7 & $12-1164$ \\
\hline Total & 48 & 66 & 114 & \\
\hline
\end{tabular}

${ }^{\top}$ Género sin epíteto especifico.

(C. martinezii y C. radicans) (GBIF, 2018). Tras relacionar la Figura 1A con 1B, se encontró que C. moschata se distribuye principalmente a lo largo de la Llanura Costera del Pacífico y Eje Neovolcánico, donde es común su cultivo; la amplia distribución que mostró esta especie concuerda con lo mencionado por Canul et al. (2005), lo cual podría explicarse por una mayor capacidad de adaptación relacionada con su amplia variación genotípica.

Con respecto a C. argyrosperma, su distribución estuvo comprendida en las regiones de la Sierra Madre del Sur y Eje Neovolcánico, mostró el mayor intervalo altitudinal (71925 msnm), aunque en menor superficie, donde se cultiva principalmente para la obtención de semilla. En la región de la Sierra Madre Occidental, sobre todo en las mayores altitudes (1705-2117 msnm), es común la presencia de $C$. pepo y C. ficifolia, estas especies comparten espacio con C. argyrosperma, pero en los límites de altura inferiores. Con relación a sus parientes silvestres (C. martinezii, C. radicans y $C$. sp.) se registró su presencia en el Eje Neovolcánico y Sierra Madre del Sur.

\section{Modelación de la distribución potencial}

Con base en los resultados obtenidos a partir de los datos de georreferenciación de presencia de especies y variables bioclimáticas, se generaron perfiles de distribución geográfica donde se determinó la probabilidad de presencia de especies de calabaza. En la Figura 2 ( A, B, C, D, E y F) se pueden observar los puntos de presencia real y las celdas con valores cercanos a 1, que corresponden a la mayor probabilidad de encontrar estas especies en el estado de Nayarit, ya que pueden ser genotipos con características valiosas para los programas de mejoramiento genético y de conservación de este grupo de especies.

En México se han realizado estudios de distribución potencial de estas especies; Mera et al. (2011) realizaron este estudio a nivel nacional; sin embargo, sus resultados no concuerdan con la distribución natural encontrada en el estado de Nayarit, ya que reportaron una sola especie ( $C$. moschata); por otra parte, Hernández-Galeno et al. (2015) estudiaron esta distribución en el estado de Guerrero y mencionan las mismas especies cultivadas de Cucurbita spp. que las encontradas en este estudio.

\section{Distribución ecofisiográfica}

Los tres primeros componentes principales (CP) explicaron el $77 \%$ de la variabilidad total; el CP1 fue el más importante al explicar $47.7 \%$ de la variación, el CP2 explicó $20.7 \%$ y el tercer componente contribuyó con 8.6 \%. En la Figura 3 se presenta en forma gráfica la dispersión de las especies con base en los dos primeros componentes principales, donde el CP1 se asoció con las variables altitud (msnm), temperaturas $\left({ }^{\circ} \mathrm{C}\right)$ de los 12 meses del año (máxima, media y mínima) y con Bio_6, que se relaciona con la temperatura mínima del mes más frío del año. Con respecto a altitud y temperatura, existe una relación entre estas dos variables, ya que se obtuvo un valor de correlación negativo (t_max: $r=-0.94, P \leq 0.01$; t_min: $r=-0.97, P \leq$ 0.01 ; t_mean: $r=-0.99, P \leq 0.01)$, por lo que a mayor altitud la temperatura decrece, lo que influye en la distribución de estas especies. En la distribución gráfica $C$. moschata y $C$. argyrosperma se ubicaron en el extremo positivo, las cuales prosperan en bajas altitudes y altas temperaturas; mientras que, en el extremo negativo se ubicaron las especies C. pepo y C. ficifolia, las cuales prosperan en las mayores altitudes y bajas temperaturas; al respecto, Espinosa et al. (2018) encontraron resultados similares en la influencia de estos factores sobre la distribución de tipos de papaya (Carica papaya L.) silvestres y cultivados.

El CP2 tuvo relación con los mayores valores de velocidad del viento en los 12 meses del año (wind_1-12), correlacionó positivamente con precipitación pluvial ( $\mathrm{mm}$ ) en los meses 


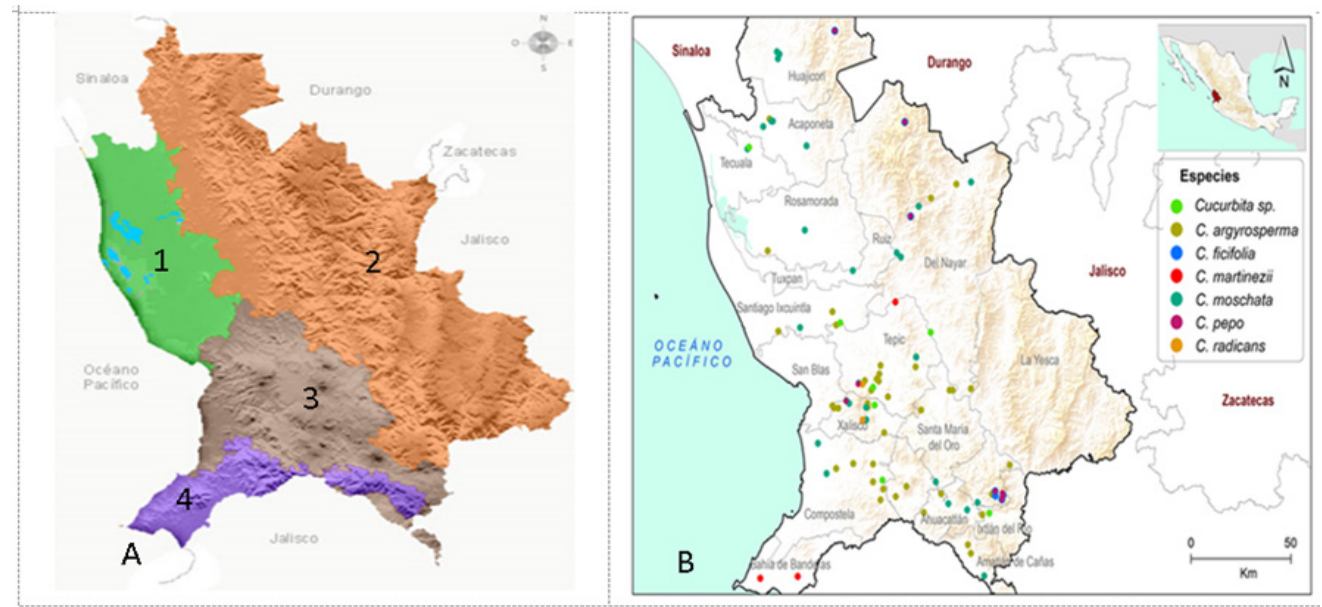

Figura 1. A). Ubicación de las provincias fisiográficas de Nayarit: 1) Llanura Costera del Pacífico, 2) Sierra Madre Occidental, 3) Eje Neovolcánico, 4) Sierra Madre del Sur (INEGI, 2000) B). Distribución geográfica actual del género Cucurbita con base en puntos de presencia en Nayarit, México.

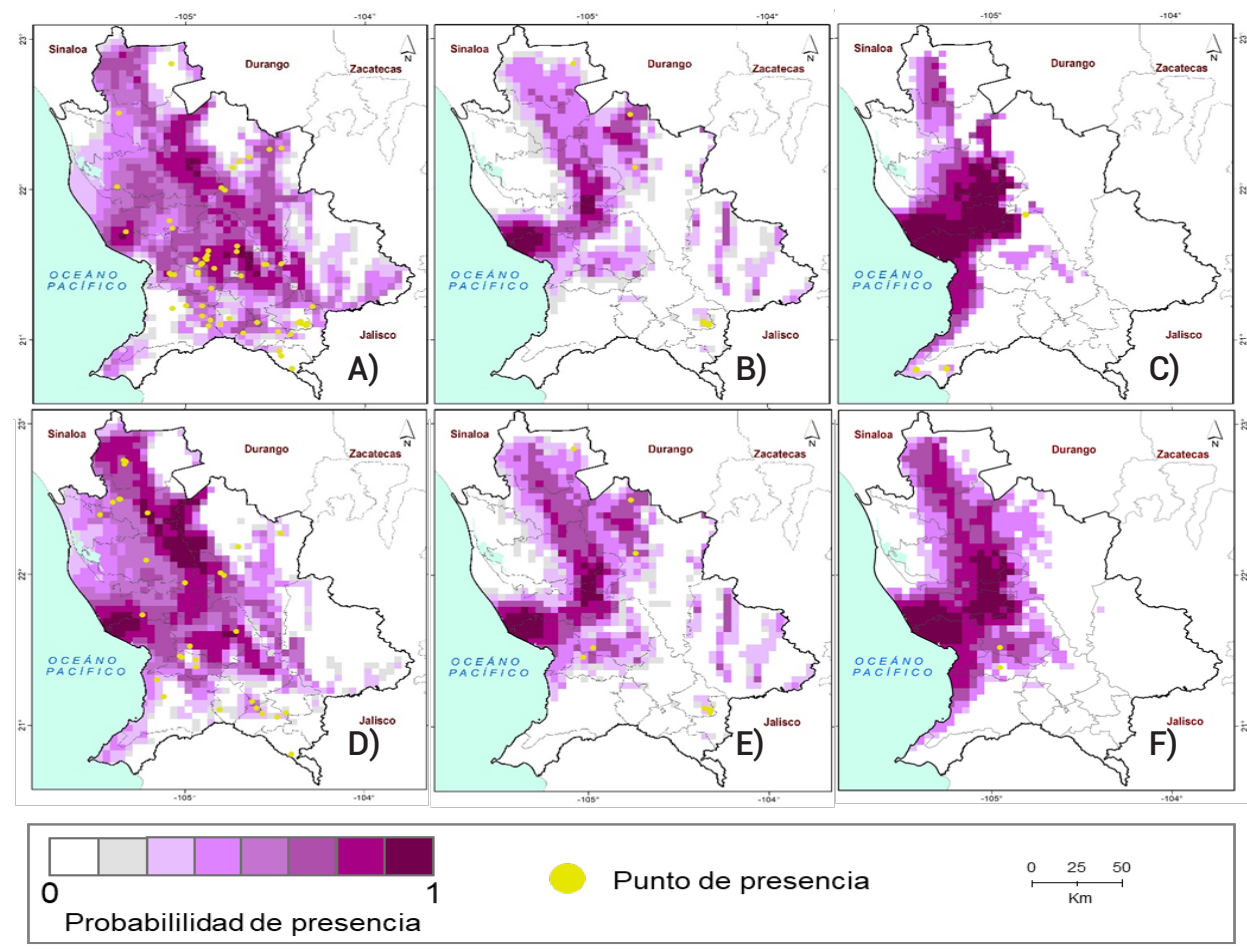

Figura 2. Distribución potencial: A) C. argyrosperma, B) C. ficifolia, C) C. martinezii, D) C. moschata, E) C. pepo y F) C. radicans en el estado de Nayarit, México.

de mayor intensidad, como son agosto $(r=0.58, P \leq 0.01)$, septiembre $(r=0.66, P \leq 0.01)$, octubre $(r=0.62, P \leq 0.01)$, noviembre $(r=0.39, P \leq 0.01)$ y diciembre $(r=0.37, P \leq$ 0.01 ), lo que indica que en aquellas regiones donde las lluvias son más frecuentes e intensas, los vientos también presentan este incremento; estas variables se localizaron en el cuadrante negativo del eje y se relacionaron con la presencia de $C$. moschata. Al respecto, como lo mencionó
Golberg (2010), la acción de los vientos también tiene un efecto sobre la temperatura foliar, transpiración y fotosíntesis, por lo que estos factores también tienen influencia en la distribución de esta especie. También se expresaron altos valores en las variables Bio_12, Bio_14, Bio_16, mismas que tienen relación con la precipitación, así como con características de suelo (arcilla, arena, profundidad, pH). El CP3 se relacionó con precipitación de 


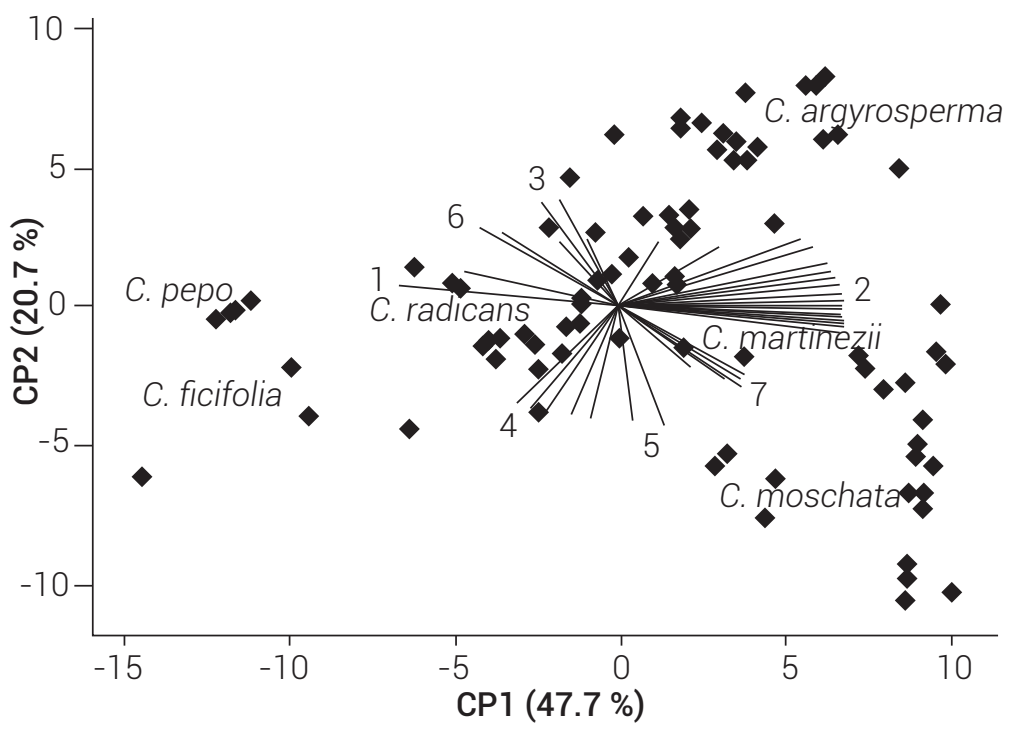

Figura 3. Dispersión de especies de calabaza, sentido y magnitud de vectores característicos con base en dos componentes principales. Variables: 1. altitud, 2. temperatura (máxima, mínima y media), 3. radiación solar, 4. precipitación, 5. dirección del viento, 6. bioclimáticas (temperatura y precipitación), 7. suelo (textura, profundidad y pH).

los tres meses más fríos del año (Bio_19). Con respecto a C. argyrosperma, ésta se ubicó en el cuadrante del eje positivo y tuvo relación con la variable de radiación solar. En lo que respecta a las especies silvestres C. radicans, C. martinezii y C. sp. éstas se ubicaron en la parte central del cuadrante, lo que sugiere que comparten condiciones ecofisiográficas similares.

En el análisis de conglomerados los registros de especies consideradas en este estudio se dividieron en tres grupos climáticos (Figura 4) que presentaron distribuciones y climas diferentes, debido a las condiciones propias de las regiones fisiográficas del estado. Los climatogramas mostraron que éstos presentan patrones climáticos distintos en los tres grupos (Figura 5).

En el Grupo I (Figura 4), predomina C. argyrosperma y en menor número $C$. moschata, las cuales se ubicaron en las regiones fisiográficas del Eje Neovolcánico y Sierra Madre del Sur, a una altitud promedio de sitios de accesiones de 889 msnm, con una precipitación anual de 1062.9 mm, el mes más seco es abril con $3.6 \mathrm{~mm}$ y el más húmedo agosto con $254.1 \mathrm{~mm}$. La temperatura media anual es de $22.24^{\circ} \mathrm{C}$ con temperaturas extremas de $10.2^{\circ} \mathrm{C}$ en el mes de enero y $32.9^{\circ} \mathrm{C}$ en el mes de mayo. De acuerdo con la clasificación de Köppen modificada por García (2004), éste corresponde a un clima semicálido subhúmedo $(A) C\left(W_{2}\right)$ (w) y cálido subhúmedo $A w_{1}(W),(A) C\left(W_{1}\right) W_{1}$ ambos con lluvias en verano.

Con respecto al Grupo II (Figura 4), las calabazas con mayor presencia corresponden a C. moschata, aunque se tienen registro de $C$. argyrosperma pero en menor número, esta ubicación corresponde a la región fisiográfica Llanura Costera del Pacífico, cuenta con una altitud promedio de sitios de localización de 126 msnm, presenta una precipitación anual de 1432.4 mm, el mes más seco es abril con $1.8 \mathrm{~mm}$ y el más húmedo agosto con $395 \mathrm{~mm}$. La temperatura media anual es de $24.7^{\circ} \mathrm{C}$, con temperaturas extremas de $14{ }^{\circ} \mathrm{C}$ en el mes de enero y de $32.4^{\circ} \mathrm{C}$ en el mes de agosto. De acuerdo con la clasificación climática de Köppen, modificada por García (2004), corresponde a un clima cálido subhúmedo con lluvias en verano, con clasificación $A w_{2}(w)$ y Aw $(w)$.

El Grupo III (Figura 4) se caracterizó por la ubicación de especies en la región fisiográfica Sierra Madre Occidental, en el cual sobresalen C. ficifolia y C. pepo. De acuerdo con los sitios de ubicación, se cuenta con una altitud promedio de 1809 msnm, la precipitación anual es de 970 mm, el mes más seco es abril, con una precipitación de $3.2 \mathrm{~mm}$ y el mes más húmedo julio con $241.9 \mathrm{~mm}$. Presenta una temperatura media anual de $17{ }^{\circ} \mathrm{C}$; la temperatura más alta es de $26.9^{\circ} \mathrm{C}$ en el mes de junio y la más baja en el mes de enero con $5.7^{\circ} \mathrm{C}$. El clima que prevalece en esta región, de acuerdo con el sistema de clasificación de Köppen modificada por García (2004), es $\mathrm{CW}_{2}(\mathrm{~W})$ y se clasifica como templado subhúmedo con lluvias en verano.

Con respecto a la información mostrada en los climatogramas (Figura 5), en la comparación de los grupos, el Grupo III presentó las más bajas temperaturas (Figura $5 F)$ y las más altas fueron para el Grupo II (Figura 5B). En cuanto a la precipitación pluvial los registros más altos 


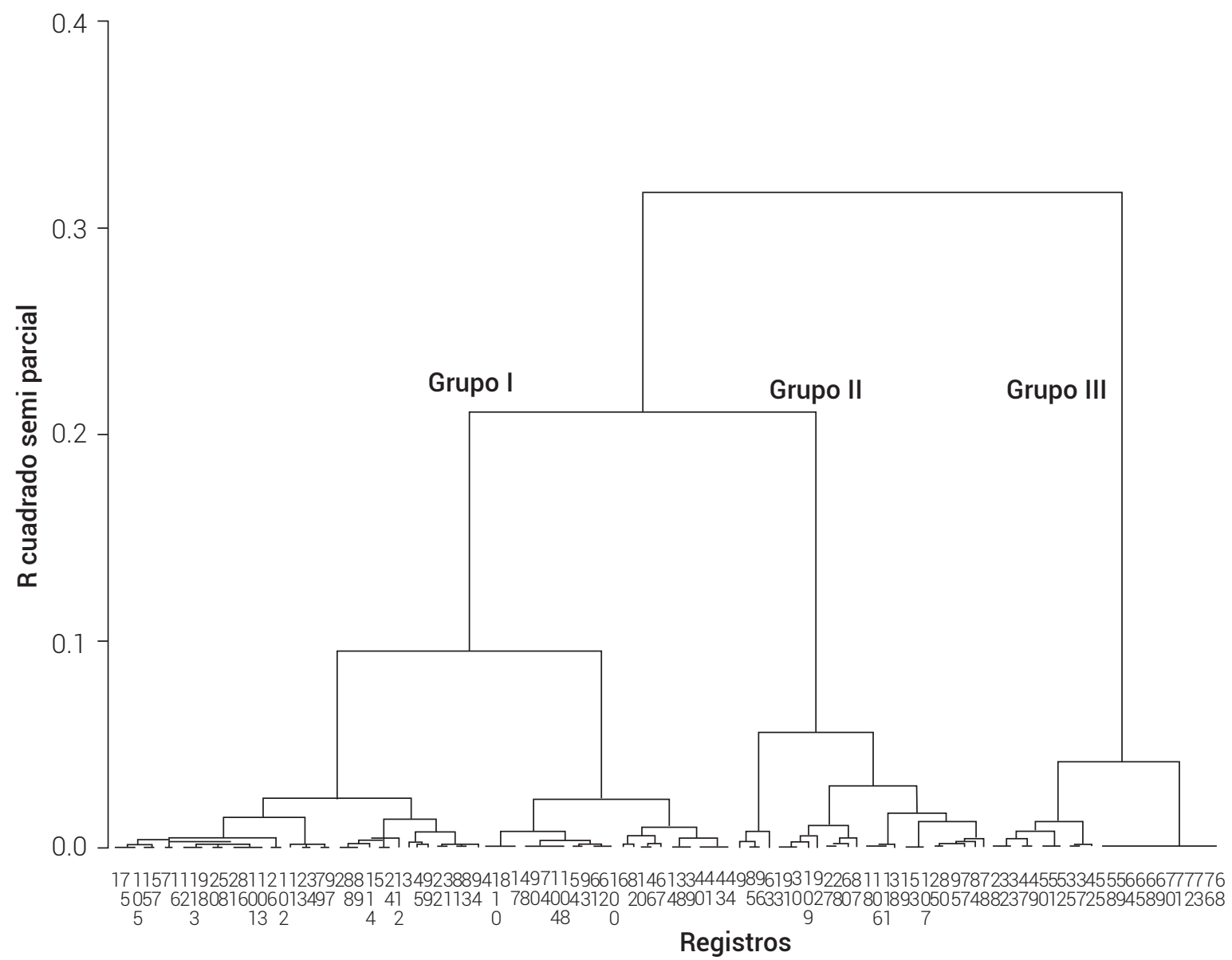

Figura 4. Dendrograma generado por 110 variables (bioclimáticas, edáficas y geofísicas) y 114 sitios geográficos con presencia de Cucurbita spp. en el estado de Nayarit, México.

fueron para el Grupo II (Figura 5C) y los menores valores para el Grupo III (Figura 5E).

En México se ha realizado este tipo de estudios de caracterización utilizando variables climáticas por NúñezColín et al. (2017) en zapote mamey (Pouteria sapota) y por Joaquín et al. (2007) en el árbol de chinene (Persea schiedeana Nees.) en el estado de Veracruz.

La información de los sitios con registros de presencia de Cucurbita spp. permitió establecer la distribución actual, potencial y ecofisiográfica de las especies incluidas en este estudio en el estado de Nayarit. Esta información permitirá ubicar áreas con probable presencia para exploraciones más dirigidas, conocer las condiciones ambientales en que se encuentran y ser utilizada en futuros programas de conservación de las especies estudiadas.

\section{BIBLIOGRAFÍA}

Canul K. J., P. Ramírez V., F. Castillo G. y J. L. Chávez S. (2005) Diversidad morfológica de calabaza cultivada en el centro-oriente de Yucatán, México. Revista Fitotecnia Mexicana 28:339-349.

Espinosa T. E., A. J. Gámez V., M Á. Ávila P., F. Palemón A. y J. Hernández R. (2018) Distribución geográfica potencial de papaya silvestre cultivada en México. Revista Mexicana de Ciencias Agrícolas 9:1377-1388, https://doi.org/10.29312/remexca.v9i7.550

ESRI, Environmental Systems Research Institute (2014) ArcGIS Desktop: Release 10.2.2. Environmental Systems Research Institute. Redlands, CA, USA. http://www.esri.com/software/arcgis/ arcgis-for-desktop (April 2018)

GBIF, Global Biodiversity Information Facility (2018) Search species. GBIF Secretariat. Copenhagen, Denmark. https://www.gbif.org/ species/search (July 2020).

García E. (2004) Modificaciones al Sistema de Clasificación Climática de Köppen. Quinta edición. Instituto de Geografía, Universidad Nacional Autónoma de México. México D. F. 98 p.

Golberg A. D. (2010) El viento y la vida de las plantas. Revista de la Facultad de Ciencias Agrarias. UNCuyo 42:221-243.

Guarino L., A. Jarvis, R. Hijmans and N. Maxted (2002) Geographic information systems (GIS) and the conservation and use of plant genetic resources. In: Managing Plant Genetic Diversity, J. M. M. Engels, V. Ramanatha Rao, A. H. D. Brown and M. T. Jackson (eds.). CABI Publishing. Wallingford, UK. pp: 387-404.

Hernández-Galeno C. A., M. Flores-Zárate, D. Castro-Lara, K. S. Vera-Sánchez y R. Toledo-Aguilar (2015) Diversidad y distribución de calabazas 

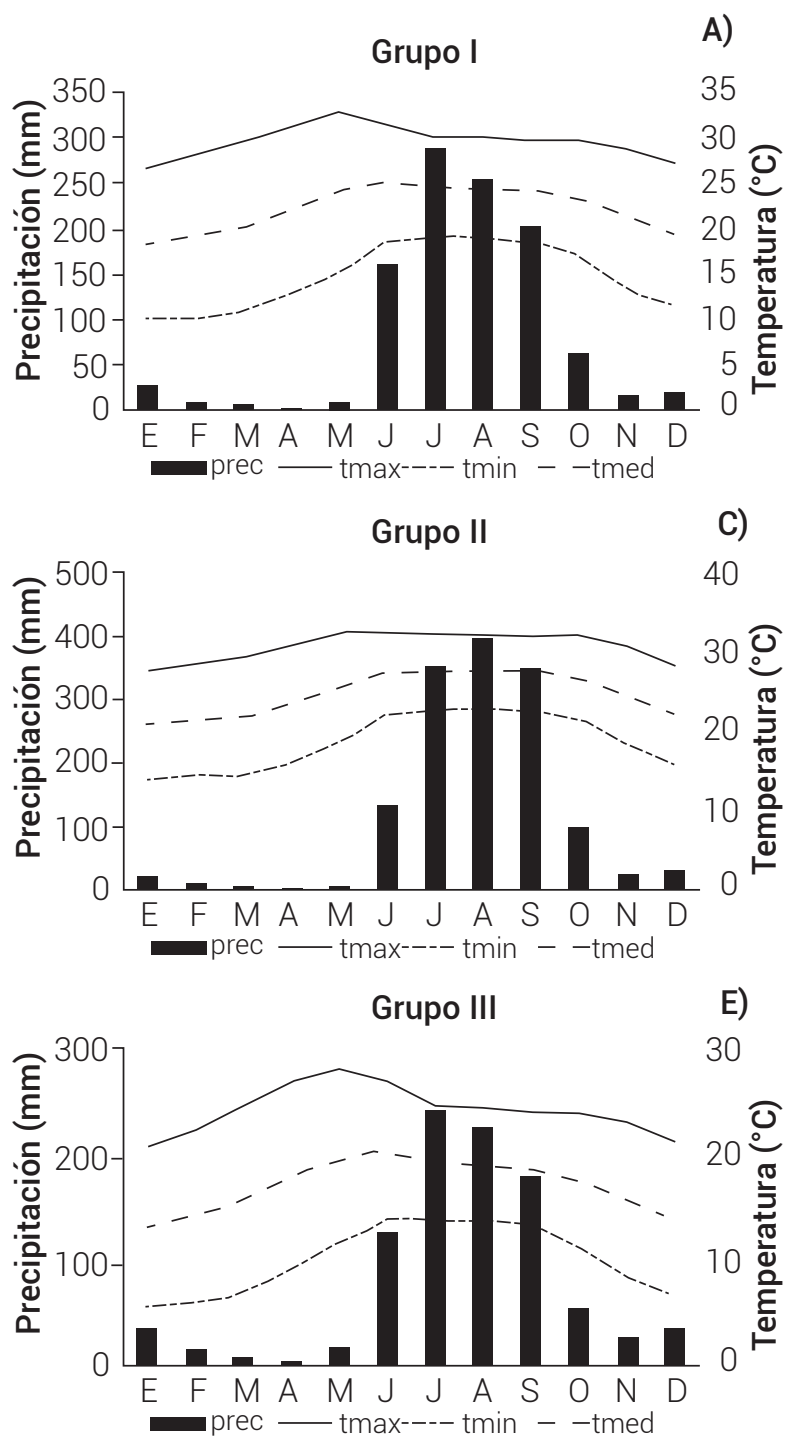

Temperatura máxima

B)

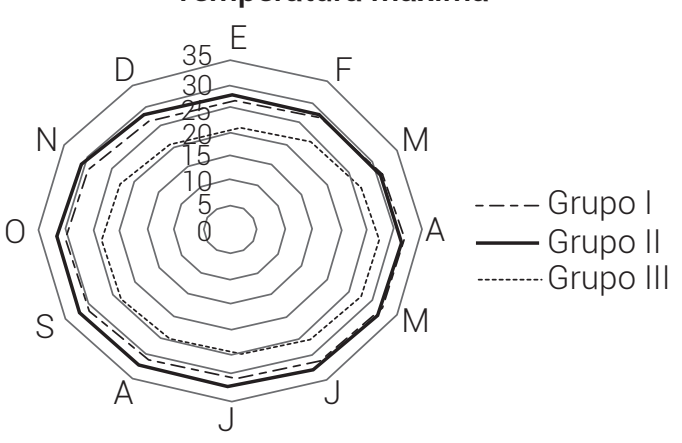

Temperatura media

D)

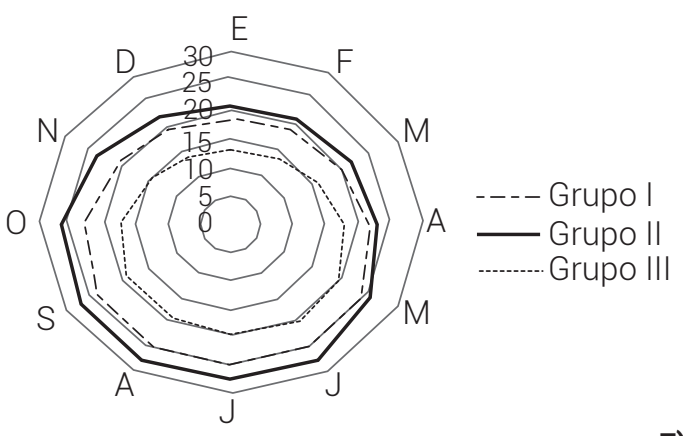

F)

Temperatura mínima

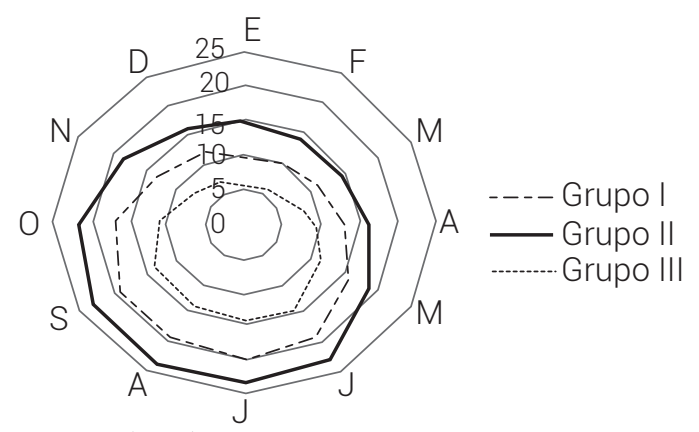

Figura 5. Climatogramas con base en temperaturas $\left({ }^{\circ} \mathrm{C}\right)$ y precipitación $(\mathrm{mm})$ de los tres grupos ecogeográficos donde se distribuyen especies de calabaza (Cucurbita spp.) en el estado de Nayarit, México. Grupo I (A y B), Grupo II (C y D), Grupo III (E y F).

(Cucurbita spp.) en Guerrero, México. Agroproductividad 8:40-46. INEGI, Instituto Nacional de Estadística Geografía e Informática (2000) Síntesis de información geográfica del estado de Nayarit. 3. Fisiografía. Instituto Nacional de Estadística Geografía e Informática. Aguascalientes, México. http:// internet.contenidos.inegi.org.mx/contenidos/productos/ prod_serv/contenidos/espanol/bvinegi/productos/ historicos/2104/702825223458/702825223458_3.pdf (Agosto 2018).

Joaquín M. M. C., J. G. Cruz C., J. De la Cruz M. y 0. Del Ángel C. (2007) Distribución ecogeográfica y características del fruto de Persea schiedeana Nees. en los Tuxtlas, Veracruz, México. Revista Fitotecnia Mexicana 30:403-410.

Maciel-Mata C. A., N. Manríquez-Morán, P. Octavio-Aguilar y G. Sánchez-Rojas (2015) El área de distribución de las especies: revisión del concepto. Acta Universitaria 25:319, https://doi.org/10.15174/au.2015.690

Mastretta-Yanes A., M. R. Bellon, F. Acevedo, C. Burgeff, D. Piñero y J. Sarukhán (2019) Un programa para México de conservación y uso de la diversidad genética de las plantas domesticadas y sus parientes silvestres. Revista Fitotecnia Mexicana 42:321334, https://doi.org/10.35196/rfm.2019.4.321-334

Mateo R. G., A. M. Felicísimo y J. Muñoz (2011) Modelos de distribución de especies: una revisión sintética. Revista Chilena de Historia Natural 84:217-240, https://doi.org/10.4067/S0716-078X2011000200008

Mera O. L. M., R. A. Bye B., C. Villanueva V. y A. Luna M. (2011) Documento de Diagnóstico de las Especies Cultivadas de Cucurbita L. Universidad Naciona Autónoma de México. México D. F. 79 p.

Minitab (2007) Meet Minitab 15 for Windows ${ }^{\circledR}$. Minitab ${ }^{\circledR}$ Inc. State College, Pennsylvania, USA. $140 \mathrm{p}$.

Núñez-Colín C. A., I. Alia-Tejacal, J. M. Villareal-Fuentes, D. Escobedo-López, J. R. Rodríguez-Núñez y V. Peña-Caballero (2017) Distribución, caracterización eco-climática y zonas potenciales de cultivo del zapote mamey en México. Revista Chapingo Serie Horticultura 23:75-88, https://doi.org/10.5154/r.rchsh.2016.05.014

Parra-Quijano M., E. Torres-Lamas, J. M. Iriondo-Alegría, F. López and A. Molina-Pertínez (2015) CAPFITOGEN Tools. User Manual Version 2.0. International Treaty on Plant Genetic Resources for Food and Agriculture. FAO. Rome. 251 p. 
Ruelas H. P. G., J. A. Aguilar C., J. D. García P., R. Valdivia B. y G. G. López G. (2015) Diversidad morfológica de especies cultivadas de calabaza (Cucurbita spp.) en el estado de Nayarit. Revista Mexicana de Ciencias Agrícolas 6:18451856, https://doi.org/10.29312/remexca.v6i8.500

SAS Institute (1999) Statistical Analysis System User' Guide Statistics. SAS Institute Inc. Cary, North Carolina, USA. 2552 p.

Scheldeman X. and M. van Zonneveld (2010) Training Manual on Spatial Analysis of Plant Diversity and Distribution. Bioversity International. Rome, Italy. 179 p.
Seoane J. y J. Bustamante (2001) Modelos predictivos de la distribución de especies: una revisión de sus limitaciones. Ecología 15:9-21.

Smith B. D. (1997) The initial domestication of Cucurbita pepo in the Americas 10,000 years ago. Science 276: 932-934 https://doi.org/10.1126/science.276.5314.932

Villanueva V. C. (2007) Calabazas Cultivadas: Identificación de Especies, Caracterización y Descripción Varietal. Universidad Autónoma Chapingo. Chapingo, Estado de México. 123 p.

Whitaker T. W. and G. W. Bohn (1950) The taxonomy, genetics, production and uses of the cultivated species of Cucurbita. Economic Botany 4:52-81, https://doi.org/10.1007/BF02859240 